\title{
FGF-2 regulates neurogenesis and degeneration in the dentate gyrus after traumatic brain injury in mice
}

\author{
Shinichi Yoshimura, ${ }^{1}$ Tetsuyuki Teramoto, ${ }^{1}$ Michael J. Whalen, ${ }^{1}$ Michael C. Irizarry, ${ }^{2}$ \\ Yasushi Takagi, ${ }^{1}$ Jianhua Qiu, ${ }^{1}$ Jun Harada, ${ }^{1}$ Christian Waeber, ${ }^{1}$ Xandra O. Breakefield, ${ }^{3}$ \\ and Michael A. Moskowitz ${ }^{1}$
}

${ }^{1}$ Neuroscience Center, Radiology Department,

${ }^{2}$ Alzheimer Disease Research Unit, Centers for Aging, Genetics, and Neurodegeneration, and

${ }^{3}$ Molecular Neurogenetics Unit, Department of Neurology, Massachusetts General Hospital and Neuroscience Program,

Harvard Medical School, Boston, Massachusetts, USA

\begin{abstract}
We studied the role of FGF-2 on regulation of neurogenesis and cell loss in the granule cell layer (GCL) of the hippocampal dentate gyrus after experimental traumatic brain injury (TBI). In both $F G F-2^{-/-}$and $F G F-2^{+/+}$mice subjected to controlled cortical impact, the number of dividing cells labeled with BrdU, injected on posttrauma days 6 through 8 , increased at 9 days after TBI, and the number of BrdU-positive cells colabeled with neuron-specific nuclear antigen significantly increased at 35 days. However, in injured $F G F-2^{-/-}$mice, BrdU-positive cells and BrdU-positive neurons (days 9, $35)$ were fewer compared with $\mathrm{FGF}-2^{+/+}$mice. There was also a decrease in the volume of the GCL and the number of GCL neurons after TBI in both $F G F-2^{-/-}$and $F G F-2^{+/+}$mice, but the decrease in both was greater in $\mathrm{FGF}_{-2 /-}$ mice at 35 days. Overexpression of FGF-2 by intracerebral injection of herpes simplex virus- 1 amplicon vectors encoding this factor increased numbers of dividing cells (day 9 ) and BrdU-positive neurons (day 35) significantly in C57BL/6 mice. Furthermore, the decrease in GCL volume was also attenuated. These results suggest that FGF-2 upregulates neurogenesis and protects neurons against degeneration in the adult hippocampus after TBI, and that FGF-2 supplementation via gene transfer can reduce GCL degeneration after TBI.
\end{abstract}

J. Clin. Invest. 112:1202-1210 (2003). doi:10.1172/JCI200316618.

\section{Introduction}

It has long been believed that the brain regenerates poorly after injury. However, this idea has been challenged by recent data showing that mammalian neural progenitor cells can proliferate and differentiate into neurons in the adult brain (1-7). Neurogenesis in the mature nervous system is subject to physiologic and pathophysiologic regulation. For example, neural progenitor cells in the subgranular zone (SGZ) of the hippocampal dentate gyrus (DG) can divide and differentiate into neurons after brain injury $(8-15)$. However, what initiates and promotes this potentially therapeutic response in vivo is still unknown. Among growth

Received for publication August 8, 2002, and accepted in revised form August 5, 2003.

Address correspondence to: Michael A. Moskowitz, Massachusetts General Hospital Building 149, 13th Street, Room 6403, Charlestown, Massachusetts 02129, USA. Phone: (617) 726-8442; Fax: (617) 726-2547;

E-mail: Moskowitz@helix.mgh.harvard.edu.

Shinichi Yoshimura and Tetsuyuki Teramoto contributed equally to this work.

Conflict of interest: The authors have declared that no conflict of interest exists.

Nonstandard abbreviations used: subgranular zone (SGZ); dentate gyrus (DG); traumatic brain injury (TBI); controlled cortical impact (CCI); granule cell layer (GCL); neuron-specific nuclear antigen (NeuN); herpes simplex virus-1 (HSV-1); enzyme immunoassay (EIA); mouse FGF-2 (mFGF-2); immediate early (IE); transducing units (TU). factors and neurotrophins that have been implicated, FGF-2 is regarded as one of the most potent (16-21). Hippocampal neural progenitor cells in culture proliferate with only FGF-2 supplementation $(17,19,20,22)$. FGF-2 and its family of receptors are widely distributed in the adult CNS (23-25), and FGF-2 is released from cells in response to injury (26). We have recently demonstrated that FGF-2 is critical for upregulation of neurogenesis in the adult DG after kainate-induced seizures and focal cerebral ischemia (15). In addition, FGF-2 has been shown to reduce neuronal death after traumatic brain injury (TBI), cerebral ischemia, and seizures (27-30). Recently, it was reported that intraventricular infusion of growth factors markedly augments endogenous progenitor proliferation, leading to regeneration of CA1 neurons and amelioration of neurologic deficits, after experimental cerebral ischemia (31). The regulation and manipulation of neurogenesis and neurodegeneration via growth factor signaling has broad implications for both the development of therapeutic strategies for repair of CNS injury after trauma, and for advancing our understanding of basic cellular mechanisms of regeneration from brain injury.

In the present study, we used a controlled cortical impact (CCI) model of TBI to evaluate the contribution of FGF-2 to cell loss and cell addition in the granule cell layer (GCL) of DG after brain injury. This model manifests progressive cell loss in the GCL as well as injured cortex (32-35). Cell loss in the GCL is assumed to 
contribute to the decrease in total volume of the GCL after injury (9). We evaluated the effect of endogenously generated FGF-2 on neuronal regeneration, neuronal loss and GCL volume loss in the DG after TBI using mice genetically deficient in FGF-2. The extent of neural progenitor cell proliferation was monitored using BrdU incorporation into replicating DNA, and differentiation of newly born cells into neurons, using immunocytochemical colocalization of a neuronal marker, neuron-specific nuclear antigen $(\mathrm{NeuN})$, with BrdU. We further evaluated the effect of overexpression of FGF- 2 by a herpes simplex virus-1 (HSV-1) amplicon vector on neurogenesis after TBI in C57BL/ 6 mice, and assessed the impact of overexpressed FGF- 2 on volume loss in the GCL after TBI.

\section{Methods}

Animals. Animal care and experimental protocols complied with the NIH's Guide for the Care and Use of Laboratory Animals (National Institutes of Health publication 86-23). FGF-2 $\mathrm{KO}$ mutant mice ( $F G F-2^{-/-}$ mice) and WT littermates $\left(F G F-2^{+/+}\right.$mice) were generated from two heterozygous mating pairs $\left(F G F-2^{+-}\right.$, SV129/Black Swiss background) (generously provided by Thomas Doetschman, University of Cincinnati College of Medicine, Cincinnati, Ohio, USA) (36). Mice were genotyped by PCR using primers specific for the WT and the FGF-2 KO alleles. Male FGF-2-/mice and $\mathrm{FGF}_{-2} 2^{+/+}$littermate controls were used at 8 to 10 weeks of age. For gene transfer experiments we used 9-week-old male C57BL/6 mice obtained from Charles River Laboratories (Wilmington, Massachusetts, USA) as WT animals for two reasons. First, we found that C57BL/6 mice robustly expressed GFP using HSV amplicon-mediated gene transfer, whereas this was less so in SV129/Black Swiss. Second, we developed a highly reproducible brain injury model in C57BL $/ 6$ mice in which $40 \%$ to $50 \%$ of cells were lost in the medial segment of the upper leaf of the DG. Because strain differences can affect hippocampal vulnerability to excitotoxicity as well as proliferation of progenitor cells in DG $(6,37)$, we compared results between groups with the same genetic background (i.e., $\mathrm{FGF}^{-2^{-/}}$and $\mathrm{FGF}-2^{+/+}$mice, or among C57BL/6 mice injected with viral vectors).

Production of cortical impact injury. The CCI model was used as previously described with minor modifications (38). The trauma protocol was approved by the Massachusetts General Hospital Institutional Animal Care and Use Committee and complied with the NIH Guide for the Care and Use of Laboratory Animals. Mice were anesthetized with $4 \%$ isoflurane (Anaquest, Memphis, Tennessee, USA), $\mathrm{N}_{2} \mathrm{O}$, and $\mathrm{O}_{2}(2: 1)$ using a Fluotec 3 vaporizer (Colonial Medical, Amherst, New Hampshire, USA) and positioned in a stereotaxic frame and maintained on $1 \%$ to $1.5 \%$ isoflurane in $70 \% \mathrm{~N}_{2} \mathrm{O}$ and $30 \%$ $\mathrm{O}_{2}$. Body temperature was monitored with a rectal probe and maintained at $36^{\circ} \mathrm{C}$ to $38^{\circ} \mathrm{C}$ with a heating pad. A 5-mm craniotomy was made using a portable drill, and a 5-mm trephine over the left parietotemporal cortex and the bone flap was removed. Mice were then subjected to CCI using a pneumatic cylinder with a 3-mm flat-tip impounder, velocity $6 \mathrm{~m} / \mathrm{s}$, depth of 0.6 $\mathrm{mm}$, and $100 \mathrm{~ms}$ impact duration. Sham controls received craniotomy only. The bone flap was replaced, and the scalp was sutured closed. The mice were allowed to recover in room air until able to ambulate (approximately 5 minutes) and returned to their cages.

BrdU injections. Animals received intraperitoneal injections of BrdU (Sigma-Aldrich, St. Louis, Missouri, USA; $50 \mathrm{mg} / \mathrm{kg}$; dissolved in sterile PBS and $0.007 \mathrm{~N} \mathrm{HCl}$ and filtered at $0.22 \mu \mathrm{m}$ ). Twice daily injections were given on days 6 and 7, followed by a single injection on day 8 after brain injury. The animals were killed 1 day after the last BrdU injection (i.e., day 9). When specified, BrdU was given twice a day on days 13 and 14, followed by a single injection on day 15 , and the animals were killed on day 16. For histologic evaluation, the animals were perfused transcardially with $4 \%$ paraformaldehyde in PBS under deep anesthesia. For enzyme immunoassay (EIA), the brains were removed without transcardial perfusion under deep anesthesia and the hippocampi were dissected and frozen immediately at $-80^{\circ} \mathrm{C}$.

Preparation of HSV-1 amplicon vector. HSV-1 amplicon vector bearing mouse FGF-2 coding sequences was prepared as previously described (15). Briefly, the mouse FGF-2 (mFGF-2) cDNA in the plasmid pBluescript (number 63348; American Type Culture Collection, Manassas, Virginia, USA) was released by digestion with Not I/Apa I. It was then inserted into the Not I/ Apa I site of pSecTag2/Hygro B (Invitrogen, Carlsbad, California, USA), so as to add an $\mathrm{N}$ terminal secretion signal from the V-J2-V region of the mouse Ig kappachain. Next, mFGF-2 cDNA with the secretion signal was digested out from the pSecTag2/Hygro B construct with Nhe I/Xho I, and ligated between the Nhe I/ $X$ Xo I sites in the multicloning site of the HSV- 1 amplicon, pHGCX (termed here HSV-1/empty, kindly provided by Y. Saeki, Massachusetts General Hospital, Charleston, Massachusetts, USA) (39). This amplicon, pHGCX/mFGF-2 (termed here HSV-1/mFGF-2) contains both a GFP cassette driven by the HSV immediate early (IE) 4/5 promoter and the FGF-2 cassette driven by the IE human $C M V$ promoter. Helper virusfree amplicon vector stocks of HSV-1/empty and HSV-1/ mFGF-2 were prepared as previously described (40). Viral titers were determined by infecting Vero 2-2 cells, which are transduced by amplicon vectors with high efficiency, and monitoring numbers of GFP-positive cells 24 hours later. The vector stocks contained $10^{7}$ transducing units (TU) per milliliter.

Vector injection. Vector injection was performed 1 hour after brain injury in anesthetized mice placed in a stereotaxic apparatus (Stoelting, Wood Dale, Illinois, USA). The bone flap made for cortical impact injury was removed and a 26-gauge needle (10- $\mu$ l Hamilton syringe) was then inserted stereotactically into the ipsilateral hippocampus at $2.5 \mathrm{~mm}$ lateral and $2.5 \mathrm{~mm}$ caudal to 
bregma to a depth of $1.5 \mathrm{~mm}$ from the dura. One microliter of vector $\left(5 \times 10^{4} \mathrm{TU}\right)$ was injected over 10 minutes $(0.1 \mu \mathrm{l} / \mathrm{min})$ with a stereotaxic injector (Stoelting). The needle was left in place for 5 minutes and then slowly withdrawn to minimize leakage.

Immunohistochemistry. Immunohistochemistry was performed on free-floating $50-\mu \mathrm{m}$ coronal sections, as reported previously $(1,5,6,18)$. We used mouse antiBrdU (1:400; Becton Dickinson, Franklin Lakes, New Jersey, USA), or for double-labeling rat anti-BrdU ascites fluid (1:100; Harlan Sera-Lab, Loughborough, United Kingdom), and mouse anti-NeuN (1:200; Chemicon, Temecula, California, USA). The number of BrdU-labeled cells was determined by staining for BrdU using the peroxidase method (ABC system) with biotinylated horse anti-mouse IgG antibodies and diaminobenzidine as chromogen (Vector Laboratories, Burlingame, California, USA). The fluorescent secondary antibodies used were cy2-labeled anti-rat $\operatorname{IgG}$ and cy3-labeled anti-mouse IgG (1:200; Jackson ImmunoResearch, West Grove, Pennsylvania, USA).

Quantification of neurogenesis and cell loss in DG after TBI. All analyses were accomplished with stereologic counting methods. A systematic random sampling of every 12 th in a series of $50-\mu \mathrm{m}$ coronal sections was prepared from each animal and processed for immunohistochemistry and Nissl staining. The hippocampus (breg$\mathrm{ma}-1.3 \mathrm{~mm}$ to $-3.1 \mathrm{~mm}$ ) was chosen because the cortical contusion overlying this region was reproducible and consistent. BrdU-positive cells were counted in the SGZ or GCL using a $40 \times$ objective lens. The SGZ was defined as a $20-\mu \mathrm{m}$ wide zone approximately two cell bodies immediately adjacent to the hilar surface of the GCL. The areas of the GCL on each section were measured on the adjacent sections stained with cresyl violet using an MCID Imaging Research image analysis system (Imaging Research Inc., St. Catherines, Ontario, Canada). The GCL volume $\left(\mathrm{GCL}_{\mathrm{V}}\right)$ was defined as that volume of the GCL in a hemisphere within bregma -1.3 $\mathrm{mm}$ to $-3.1 \mathrm{~mm}$, and was calculated (41). The number of BrdU-positive cells within the $\mathrm{GCL}_{V}$ then was calculated by multiplying volume density $\left(\right.$ cells $/ \mathrm{mm}^{3}$ ) of BrdU-positive cells by the volume of $\mathrm{GCL}_{\mathrm{V}}$.

Counting of BrdU-positive cells coexpressing NeuN was performed using a Nikon TE 400 microscope with a laser confocal scanning system MRC 1024 (Bio-Rad, Wattford, United Kingdom). For each animal, the number of double-labeled cells was counted in the ipsilateral GCL. Cells were considered double-labeled when BrdU and NeuN immunoreactivity overlapped at five levels through a section (z-step $1 \mu \mathrm{m})$.

We determined percentage volumetric change of $\mathrm{GCL}_{V}$ in the hemisphere ipsilateral to the contusion relative to the $\mathrm{GCL}_{V}$ of the contralateral side (42). To determine whether changes in $\mathrm{GCL}_{V}$ of ipsilateral versus contralateral hemispheres reflected changes in neuron numbers, granule cells in the $\mathrm{GCL}_{V}$ were counted in $\mathrm{FGF}^{-2^{-/-}}$and $\mathrm{FGF}^{2} 2^{+/+}$mice 35 days after CCI. Granule cell counts in the $\mathrm{GCL}_{V}$ were performed in coronal $50-\mu \mathrm{m}$ sections spaced $600 \mu \mathrm{m}$ apart and stained with cresyl violet using the optical dissector technique (Bioquant Basic Stereology Toolkit; R\&M Biometrics Inc., Nashville, Tennessee, USA) $(43,44)$. The $\mathrm{GCL}_{V}$ was systematically randomly sampled with approximately 20 optical dissectors $(10 \times 10$ $\mu \mathrm{m}$ sampling box with extended exclusion lines) under $100 \times$ water objective. The coefficient of error for the counting technique was less than 0.10 (45). To estimate the total number of granule cells within the $\mathrm{GCL}_{\mathrm{V}}$ (neurons within lesion volume $\left.=G_{C L}\right)$, the resulting neuronal volume density (cells/sample volume, sample volume: $10 \times 10 \times 50 \mu \mathrm{m}^{3}$ ) was multiplied by the volume of the $\mathrm{GCL}_{\mathrm{V}}$. We determined percentage change of $\mathrm{GCL}_{\mathrm{N}}$ in the hemisphere ipsilateral to the contusion relative to the $\mathrm{GCL}_{\mathrm{N}}$ of the contralateral side.

Cerebral cortex contusion volume after CCI was measured in the same four cresyl violet-stained sections used for DG cell counts by image analysis (Imaging Research MCID image analysis system) using the Cavalieri technique (41).

EIA. Stored hippocampi at $-80^{\circ} \mathrm{C}$ were homogenized in buffer solution $(20 \mathrm{mM}$ Tris- $\mathrm{HCl}, \mathrm{pH} 8.0,137 \mathrm{mM}$ $\mathrm{NaCl}, 1 \mathrm{mM}$ DTT, $0.5 \%$ Triton X-100, $0.5 \mathrm{mM}$ PMSF) and centrifuged at $14,000 \mathrm{~g}$ for 30 minutes at $4^{\circ} \mathrm{C}$. Protein concentration of each supernatant was determined by a protein assay kit (Bio-Rad Laboratories Inc., Hercules, California, USA). EIA for FGF-2 was performed using an assay kit (Quantikine HS; R\&D Systems, Minneapolis, Minnesota, USA) according to the manufacturer's instruction. For intra- and interassay precision, coefficient variability was less than $15 \%$.

Statistical analysis. All values are expressed as the mean \pm SD. ANOVA with Bonferroni's post hoc analysis in StatView 5.0 for Macintosh (SAS Institute, Cary, North Carolina, USA) was used for statistical analysis throughout the study. $P$ values less than 0.05 were considered statistically significant.

\section{Results}

BrdU incorporation into the DG in FGF-2+/ and FGF-2-/- mice after TBI. To measure the extent of cell proliferation and the effect of FGF-2 after TBI, we measured BrdU incorporation into the nuclei of progenitor cells in SGZ of $\mathrm{FGF}_{-2} \mathrm{I}^{++}$and $\mathrm{FGF}_{-2} 2^{-/-}$mice. Few BrdU-labeled cells were present within the SGZ of the ipsilateral DG in the sham-operated $\mathrm{FGF}-2^{+/+}$and $F G F-2^{-/-}$mice (Figure 1a). The number of BrdU-positive cells did not differ between $\mathrm{FGF}_{-2} 2^{+/+}$and $\mathrm{FGF}-2^{-/-}$mice after sham operation (Figure 1b). BrdU-labeled cells increased at day 9 after TBI in both groups, but the increase at day 9 was greater in $\mathrm{FGF}^{2+/+}$ mice (4.4-fold vs. 2.9-fold in $\mathrm{FGF}^{+2^{++}}$and FGF-2-/, respectively, $P<0.05$; Figure $1 \mathrm{~b}$ ). At day 16 after TBI, the increased number of BrdU-positive cells was still significantly greater in $\mathrm{FGF}-2^{+/+}$compared with FGF-2-1- mice $(P<0.05)$. Next, FGF-2 levels were measured in the hippocampus after injury. We previously reported that the level of FGF-2 protein in the hippocampus in uninjured mice was approximately 100 $\mathrm{pg} / \mathrm{mg}$ protein in $\mathrm{FGF}^{-2^{+/+}}$animals and undetectable in 


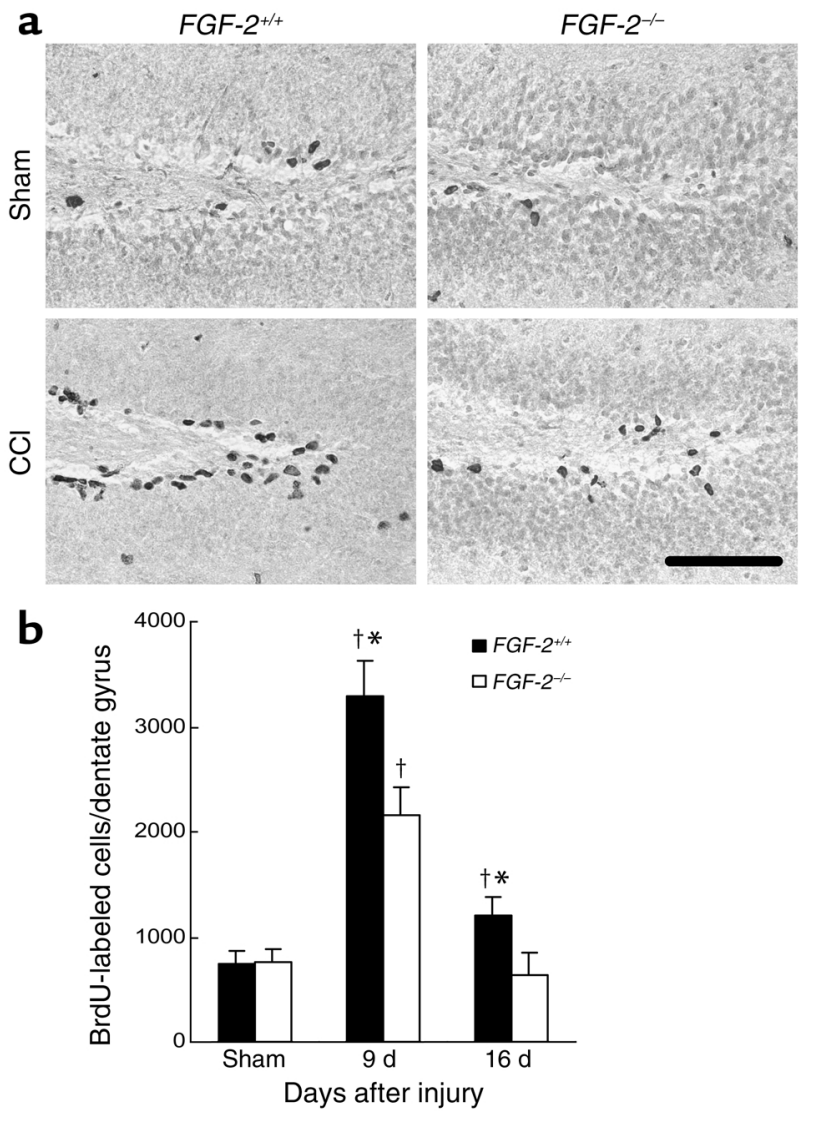

FGF-2-/- animals (15). Despite the increase in BrdU labeling, levels of FGF-2 within the hippocampus of $F G F-2^{+/+}$ mice did not change significantly after TBI (Table 1 ). These results suggest that basal levels of endogenously generated FGF-2 are sufficient to facilitate proliferation of progenitor cells after TBI. On the other hand, increased numbers of dividing cells in FGF-2-deficient mice at day 9 indicates that FGF- 2 is not the only stimulus for proliferation of progenitor cells in DG after TBI.

We also examined the number of BrdU-positive cells in the CA1 and the temporal cortex in the same specimens to examine for regional specificity. There was no significant increase in BrdU-positive cells before and at 9 days after TBI in both CA1 pyramidal layer and temporal cortex from bregma $-1.3 \mathrm{~mm}$ to $-3.1 \mathrm{~mm}$ (CA1 pyramidal layer; untreated: $8.2 \pm 4.6, \mathrm{TBI}, 10.2 \pm 5.2$, temporal cortex; untreated: $4.1 \pm 4.4$, TBI, $5.8 \pm 6.3$ cells/ $\mathrm{mm}^{2}$ ). These results suggested that neurogenesis was specifically upregulated in the GCL in the CCI model used in this study.

FGF-2 gene transfer increases FGF-2 protein in mouse hippocampus after TBI. We next examined the effect of overexpressed FGF-2 protein on proliferation of progenitor cells in the SGZ after TBI. Injection of an HSV-1 amplicon vector encoding GFP to the hippocampus of injured brain yielded robust gene expression at the injection site and within DG after 48 to 72 hours (data not shown), consistent with previous reports (15). Next, we injected the HSV-1/mFGF-2 vector encoding GFP and mouse

\section{Figure 1}

BrdU-positive cells in the SGZ of DG after CCl in $\mathrm{FGF}^{+/++}$and $\mathrm{FGF}^{-2^{-/}}$ mice. (a) To label dividing cells, BrdU was injected intraperitoneally at 6,7 , and 8 days after $\mathrm{CCl}$ or sham operation, and mice were killed on day 9 . BrdU labeling was detected by immunohistochemistry (see Methods). Few BrdU-labeled cells were present within the $\mathrm{SGZ}$ of the ipsilateral DG in the sham-operated $\mathrm{FGF}-2^{+/+}$and $\mathrm{FGF}-2^{-/-}$ mice. After $\mathrm{CCl}$, an increase in the number of positive cells was detected, but the increase was less in $\mathrm{FGF}-2^{-/-}$mice. Tissue section coordinate, bregma $-1.9 \mathrm{~mm}$. Scale bar: $100 \mu \mathrm{m}$. (b) Quantification of BrdU-positive cells in DG after CCl in $\mathrm{FGF}-2^{+/+}$and $\mathrm{FGF}-2^{-/-}$ mice. BrdU was injected at 6,7 , and 8 days, or 13,14 , and 15 days after $\mathrm{CCl}$, and animals killed on day 9 or 16, respectively. Shamoperated mice were injected with BrdU on days 6,7 , and 8 , and killed on day 9. Cell proliferation was assessed by immunohistochemical detection of incorporated $\mathrm{BrdU}$, and the positive cells were counted at four different levels of DG using stereologic methods. Data are expressed as the total number of labeled cells within SGZ (see Methods). After TBI, the number of BrdU-positive cells increased at 9 and 16 days in $\mathrm{FGF}-2^{+/+}$mice (black bars), whereas the increase in $\mathrm{FGF}_{-2-2^{--}}$(white bars) was present only at 9 days after injury. ${ }^{\dagger} P<0.05$ compared with sham-operated mice; ${ }^{*} P<0.05$, $F G F-2^{+/+}$vs. $F G F-2^{-/-} . n=4-6$ per group.

FGF-2 into the injured hippocampus 1 hour after TBI and measured FGF-2 levels in the hippocampus at day 7. The levels of FGF-2 without vector injection did not differ from those in $\mathrm{FGF}_{-2 /+}$ mice at 7 days after TBI (Table 1). On the other hand, the levels of FGF-2 increased about 2.5 -fold in ipsilateral hippocampus after HSV-1/mFGF-2 injection ( $P<0.05$; Table 1$)$. Thus, HSV$1 /$ empty vector did not perturb endogenous FGF-2 levels, and the HSV-1/mFGF-2 vector significantly increased FGF-2 protein in the hippocampus.

FGF-2 gene transfer increases the number of BrdU-positive cells in DG after TBI. To assess the effects of overexpressed FGF-2 on proliferation of progenitor cells in $\mathrm{SGZ}$, the incorporation of BrdU was examined in C57BL/6 mice injected with HSV-1/empty or HSV-1/ mFGF-2. First, the number of BrdU-positive cells in DG of the sham-operated or injured mice did not differ between those treated with and without HSV-1/ empty vector (data not shown). Next, consistent with the findings in $\mathrm{FGF}^{+2^{+/+}}$mice (Figure $1 \mathrm{~b}$ ), TBI increased the number of BrdU-positive cells by 4.3 -fold at 9 days after injury in C57BL/6 mice, compared with sham operated animals $(P<0.05$; Figure 2 , a-e). After injury and HSV-1/mFGF-2 injection, the number of BrdU-

\section{Table 1}

FGF-2 levels in hippocampus after CCl

\begin{tabular}{ccccc} 
& Untreated & Day 1 & Day 3 & Day 7 \\
FGF-2++ & $96 \pm 36$ & $121 \pm 32$ & $94 \pm 32$ & $113 \pm 36$ \\
FGF-2 ${ }^{-/}$ & undetectable & $\mathrm{np}$ & $\mathrm{np}$ & undetectable \\
& Untreated & HSV-1/empty & HSV-1/mFGF-2 \\
C57BL/6 & $109 \pm 36$ & $110 \pm 31$ & $278 \pm 93^{\mathrm{A}}$ \\
\hline
\end{tabular}

All numbers are mean $\pm \mathrm{SD}(\mathrm{pg} / \mathrm{mg}$ protein $)\left(n=4-6\right.$ per group). ${ }^{A} P<0.05 \mathrm{vs}$. HSV-1/empty measured at 7 days. np, not performed. 

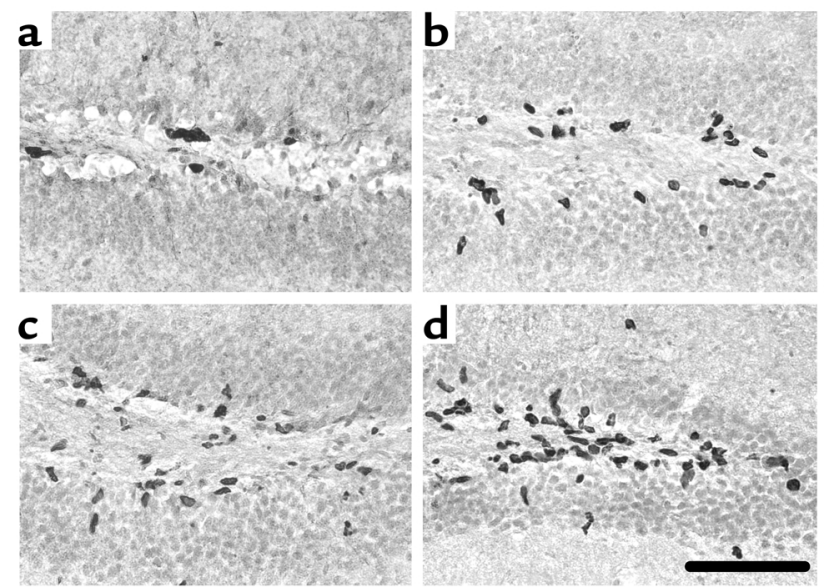

e

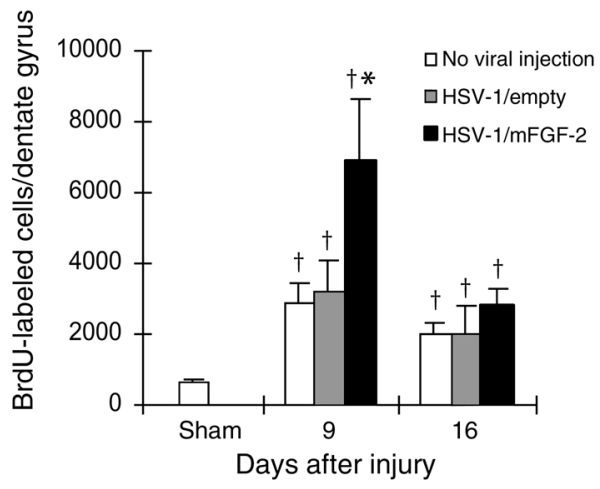

positive cells increased by 2.2 -fold compared with HSV-1/empty injection and 10.3-fold compared with sham mice $(P<0.05$; Figure $2, \mathrm{a}-\mathrm{e})$. According to our previous report, gene transfer using HSV-1/mFGF-2 vector in intact $\mathrm{FGF}-2^{+/+}$mice doubled BrdU incorporation into $S G Z$ cells relative to $H S V-1 /$ empty vector (15). These results indicate that, after TBI, FGF-2 can strongly promote progenitor cell proliferation in the SGZ. The increased rate of cell division, however, was not sustained by day 16, matching the normal response to injury (Figure $1 \mathrm{~b}$ ).

Effect of increased FGF-2 expression on neuronal differentiation in the $D G$. To assess the phenotype of newborn cells after TBI, and to determine the effect of FGF-2 expression on cell differentiation, we determined whether BrdU-positive cells expressed NeuN 35 days after TBI in $\mathrm{FGF}-2^{+/+}$and $\mathrm{FGF}-2^{-/-}$mice. Baseline numbers of BrdU/NeuN-positive cells did not differ between $\mathrm{FGF}_{-2^{+/+}}$and $\mathrm{FGF}_{-2^{-/-}}$sham-operated mice (Table 2). At 35 days after TBI, the number of $\mathrm{BrdU} / \mathrm{NeuN}$-positive cells increased by 2.8 -fold in $F G F-2^{+/+}(P<0.01$ vs. sham injured) and by 1.8 -fold in $F G F-2^{-/-}$mice $(P<0.05$ vs. sham injured). In addition, the number of BrdU/NeuN-positive cells was significantly greater in $\mathrm{FGF}-2^{+/+}$mice versus $\mathrm{FGF}-2^{-/-}$after TBI $(P<0.01$, Table 2$)$.

Because BrdU was injected into mice at a time when TUNEL staining was not observed in the DG after injury (data not shown), it is unlikely that BrdU was incorporated into injured NeuN-positive neurons dur-

\section{Figure 2}

Effect of gene transfer of FGF-2 with HSV-1 amplicon vector on proliferation of progenitor cells in SGZ after $\mathrm{CCI}$. One hour after sham operation (a) or $\mathrm{CCl}$ (panels b-d), HSV-1/mFGF-2 vector (d) or empty vector (c) was injected stereotactically into the injured hippocampus of C57BL/ 6 mice (see Methods). Mice underwent intraperitoneal injection of $\mathrm{BrdU}$ at 6,7 , and 8 days or 13,14 , and 15 after $\mathrm{CCl}$ and were killed on day 9 or day 16 (the latter not shown). BrdU staining was detected immunohistochemically as described in Figure 1. Robust BrdU labeling was noted in the SGZ of the ipsilateral DG after $\mathrm{CCl}(\mathbf{b})$ vs. sham operation (a), and after $\mathrm{CCl}$ in mice injected with $\mathrm{HSV}-1 / \mathrm{mFGF}-2$ (d) vs. control vector (c). Tissue section coordinate, bregma $-1.9 \mathrm{~mm}$. Scale bar: $100 \mu \mathrm{m}$. (e) Quantification of BrdU-positive cells in SGZ in C57BL/ 6 mice injected with HSV-1/mFGF-2 or HSV-1/empty. HSV-1/mFGF-2 or HSV-1/ empty was injected into injured hippocampus 1 hour after $\mathrm{CCl}$. BrdU was injected at 6,7 , and 8 days, or 13,14 , and 15 days after $\mathrm{CCl}$, and animals killed on day 9 or 16 , respectively. Sham-operated mice were injected with BrdU on days 6,7 , and 8 , and killed on day 9. After $\mathrm{CCl}$, mice injected with HSV-1/mFGF-2 (black bars) had a greater number of BrdU-positive cells in SGZ on day 9 vs. HSV-1/empty (gray bar) or after CCl only (white bars). ${ }^{\dagger} P<0.05$ compared with sham-control. ${ }^{*} P<0.05$ compared with HSV-1/ empty ( $n=6$ per group)

ing repair of damaged DNA. In addition, there was complete absence of BrdU/NeuN-positive cells in the GCL of $\mathrm{FGF}^{-2^{+/+}}$and $\mathrm{FGF}_{-22^{-/}}$mice at 9 days after TBI (the day after the last BrdU injection), suggesting that BrdU-labeled NeuN-positive cells are granule neurons differentiated from newly generated progenitor cells in SGZ of DG at 5 weeks.

After FGF-2 gene transfer, the number of BrdU/NeuNpositive cells increased by 2.5 -fold (Figure 3 and Table $2, P<0.01$ vs. HSV-1/empty), indicating that FGF- 2 can increase the population of neurons in GCL after TBI.

Effect of FGF-2 gene deficiency on dentate GCL volume loss after TBI. Contusion volume (primary cortical tissue loss after CCI) at the level of impact $(0.6 \mathrm{~mm})$ was limited to cortex and did not include the hippocampus. Contusion volume did not differ between littermates at 9 days after TBI $\left(2.09 \pm 1.47\right.$ in $F G F-2^{+/+}$vs. $2.03 \pm 1.23$ $\mathrm{mm}^{3}$ in $F G F-2^{-/-}$mice, $P>0.05$ ), indicating that FGF-2

Table 2

Number of BrdU/NeuN-positive cells 35 days after injury

\begin{tabular}{|c|c|c|}
\hline & Group & BrdU/NeuN-positive cells \\
\hline \multirow[t]{2}{*}{ Sham } & $F G F-2^{+/+}$ & $418 \pm 52$ \\
\hline & FGF-2-/- & $347 \pm 61$ \\
\hline \multirow[t]{2}{*}{35 days after injury } & $F G F-2^{+/+}$ & $1177 \pm 239^{A}$ \\
\hline & FGF-2-/- & $621 \pm 113^{B, C}$ \\
\hline Sham & C57BL/6 & $362 \pm 39$ \\
\hline \multirow[t]{2}{*}{35 days after injury } & HSV-1/empty & $873 \pm 272^{D}$ \\
\hline & HSV-1/mFGF-2 & $2156 \pm 417 \mathrm{E}, \mathrm{F}$ \\
\hline
\end{tabular}

All numbers are mean $\pm \mathrm{SD}$ (cells $/ \mathrm{GCL}, n=4-6$ per group). ${ }^{A} P<0.01 \mathrm{vs} .+/+$ after sham operation. ${ }^{B} P<0.05$ vs. $-/-$ after sham operation. ${ }^{C} P<0.01$ vs. $+/+$ after injury. ${ }^{\mathrm{D} P}<0.05$ vs. $\mathrm{C} 57 \mathrm{BL} / 6$ mice after sham operation. $\mathrm{E} P<0.01$ vs. C57BL/ 6 mice after sham operation. ${ }^{F} P<0.01$ vs. HSV-1/empty. Sham, 35 days after sham operation. HSV-1/empty, C57BL/ 6 mice injected with HSV-1/empty vector. HSV-1/mFGF-2, C57BL/ 6 mice injected with HSV-1/mFGF-2 vector. 

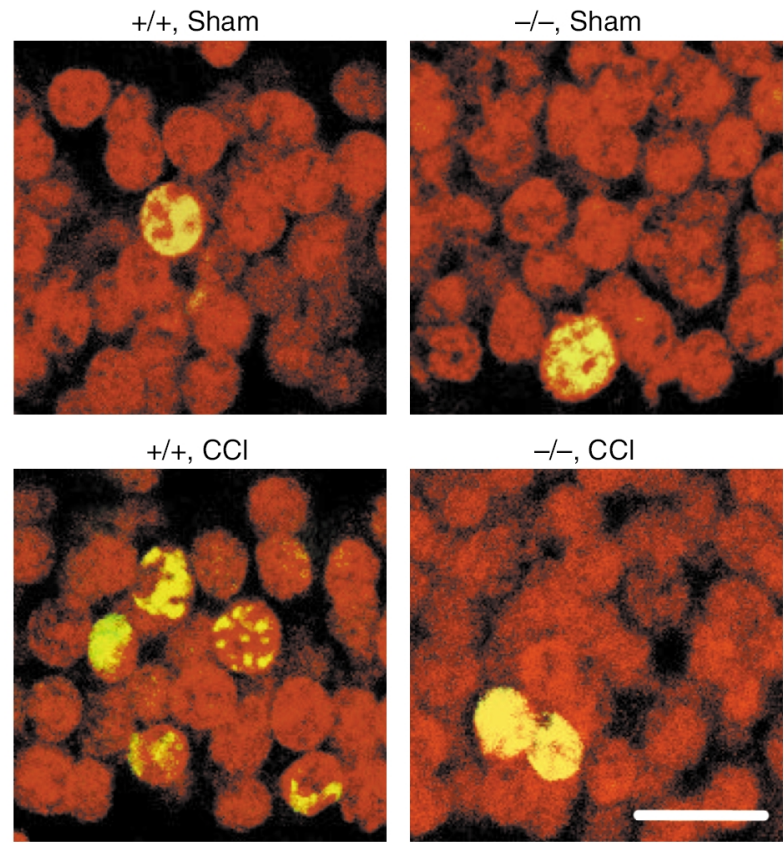

Figure 3

Differentiation of BrdU-labeled cells into neurons within the GCL of DG after TBI. BrdU was injected on days 6, 7, and 8 after $\mathrm{CCI}$ or sham operation, and mice were killed on day 35. Coronal brain sections stained for BrdU immunoreactivity (cy2, green) and the neuronal marker, NeuN (cy3, red) were examined by laser scanning confocal microscopy (see Methods). The BrdU/NeuN-positive cells (yellow nuclei) were more numerous in $\mathrm{FGF}^{-2^{+/+}}(+/+)$ than in FGF-2-/- $(-/-)$ mice (see Table 1$)$. Tissue section coordinate, bregma $-2.5 \mathrm{~mm}$. Scale bar: $20 \mu \mathrm{m}$.

deficiency did not modify posttraumatic cortical tissue loss. There was also extensive cell death (secondary GCL cell loss) in the hippocampus, including the DG after TBI $(32,46)$, which leads to volume loss in GCL $(32,42)$. We therefore investigated the effect of FGF-2 on GCL volume loss at 9 and 35 days after TBI. There was no significant difference in the estimated volume of ipsilateral GCL between sham-operated $\mathrm{FGF}^{+2^{++}}$ and $F G F-2^{-/-}$mice (data not shown). There was also no significant difference in the volume of contralateral GCL between $\mathrm{FGF}-2^{+/+}$ and FGF-2-/- mice (Table 3). Morphologic changes in the ipsilateral GCL were observed as early as 9 days after TBI, and became marked 35 days later (Figure 4a). At 9 days, the total GCL volume was decreased by $16 \%$ in both $\mathrm{FGF}^{-2^{+/+}}$and $\mathrm{FGF}-2^{-/-}$mice (Figure $4 \mathrm{~b}$ ). At this time, the superomedial part of the upper blade was thinned and the total volume was reduced as reported previously (42). On day 35 , the volume loss became more marked in $F G F-2^{-/-}$ mice $(37 \% \pm 3 \%$ reduction in mutant vs. $24 \% \pm 4 \%$ in $F G F-2^{+/+}$mice, $P<0.05$; Figure $4 \mathrm{~b}$ ). These data indicate that lack of brain FGF-2 expression contributes to volume loss in GCL after TBI.

Next, granule cells within the GCL were counted in FGF-2 $2^{+/+}$and $F G F-2^{-/-}$mice 35 days after TBI to determine whether the decrease in volume reflected cell loss. The density of cells in the ipsilateral and contralateral GCL did not differ significantly between the littermates $(3.75 \pm 0.22$ cells per sample volume in $\mathrm{FGF}^{2+/+}$ vs. $3.45 \pm 0.27$ in FGF-2-/; Table 3). However, the absolute numbers of granule cells in the ipsilateral GCL were significantly different between groups (Table 3), with percentage neuronal loss being more marked in $\mathrm{FGF}-2^{--}$mice at 35 days after injury $(48 \% \pm 3 \%$ in mutant vs. $33 \% \pm 5 \%$ in WT mice, $P<0.05$; Figure 4c). Thus, in this TBI model, the changes in the GCL volume correlated with decreased granule cell numbers. These results suggest that endogenously generated FGF-2 protects GCL neurons against long-lasting degenerative changes after TBI.

Effect of FGF-2 overexpression on GCL volume loss after TBI. We speculated that if a deficiency of FGF-2 promoted greater thinning and volume loss in GCL after injury, overexpression of FGF-2 by gene transfer technique might afford protection. There was no significant difference in neuronal density in the GCL between $\mathrm{C} 57 \mathrm{BL} / 6$ mice and $\mathrm{FGF}-2^{+/+}$mice before and after TBI in our preliminary experiments (data not shown). Then, GCL volume was measured in C57BL/6 mice with or without intraventriclar injection of HSV-1/mFGF-2 or HSV-1/empty vector after TBI. Consistent with the findings in Figure 4, b and c, in FGF-2 $2^{+/+}$mice, TBI decreased the GCL volume in C57BL/6 at 9 and 35 days after injury in injured animals with empty vector (Figure 5). At 9 days after injury, there were no significant differences in the volume loss between groups injected with FGF-2 bearing $(13 \% \pm 2 \%)$ versus empty vector $(16 \% \pm 3 \%)$ (Figure 5). However, by 35 days, GCL volume loss was almost $50 \%$ less in mice treated with HSV-1/mFGF-2 $(15 \% \pm 3 \%$ in HSV- $1 / \mathrm{mFGF}-2$ - vs. $27 \% \pm 6 \%$ in HSV- 1 / empty-treated mice, $P<0.05$, Figure 5). Thus, overexpression of FGF-2 within hippocampus reduced the progressive GCL volume loss.
Table 3

Cell counts in GCL 35 days after $\mathrm{CCl}$

FGF-2 $2^{+/+}$ $\mathrm{FGF}_{-2} \mathrm{-}^{-}$

Ipsilateral side
Volume of GCL $\left(\mu \mathrm{m}^{3}\right)$

Total number of granule cells Cell density (cells/SV)

Contralateral side

Volume of GCL $\left(\mu \mathrm{m}^{3}\right)$

Total number of granule cells

Cell density (cells/SV)

$$
\begin{gathered}
1.70 \times 10^{8} \pm 0.14 \times 10^{8 \mathrm{~A}} \\
1.27 \times 10^{5} \pm 0.10 \times 10^{5 \mathrm{~A}} \\
3.75 \pm 0.22
\end{gathered}
$$
$2.22 \times 10^{8} \pm 0.13 \times 10^{8}$ $1.89 \times 10^{5} \pm 0.14 \times 10^{5}$ $4.26 \pm 0.25$

$1.38 \times 10^{8} \pm 0.14 \times 10^{8 \mathrm{~A}, \mathrm{~B}}$ $0.95 \times 10^{5} \pm 0.08 \times 10^{5 \mathrm{~A}, \mathrm{C}}$ $3.45 \pm 0.27$

$2.18 \times 10^{8} \pm 0.14 \times 10^{8}$ $1.82 \times 10^{5} \pm 0.17 \times 10^{5}$ $4.18 \pm 0.25$
All numbers are mean \pm SD ( $n=4-6$ per group). Ipsilateral and contralateral sides are ipsilateral and contralateral $\mathrm{GCL} 35$ days after $\mathrm{CCl}$ in $F G F-2^{+/+}$and $F G F-2^{-/-}$mice. ${ }^{A} P<0.05$ vs. contralateral side in each littermate. ${ }^{B} P<0.05$ vs. ipsilateral side in $F G F-2^{+/+}$mice. ${ }^{C} P<0.01$ vs. ipsilateral side in FGF-2+/+ mice. SV, sample volume $\left(5,000 \mu \mathrm{m}^{3}\right)$. 

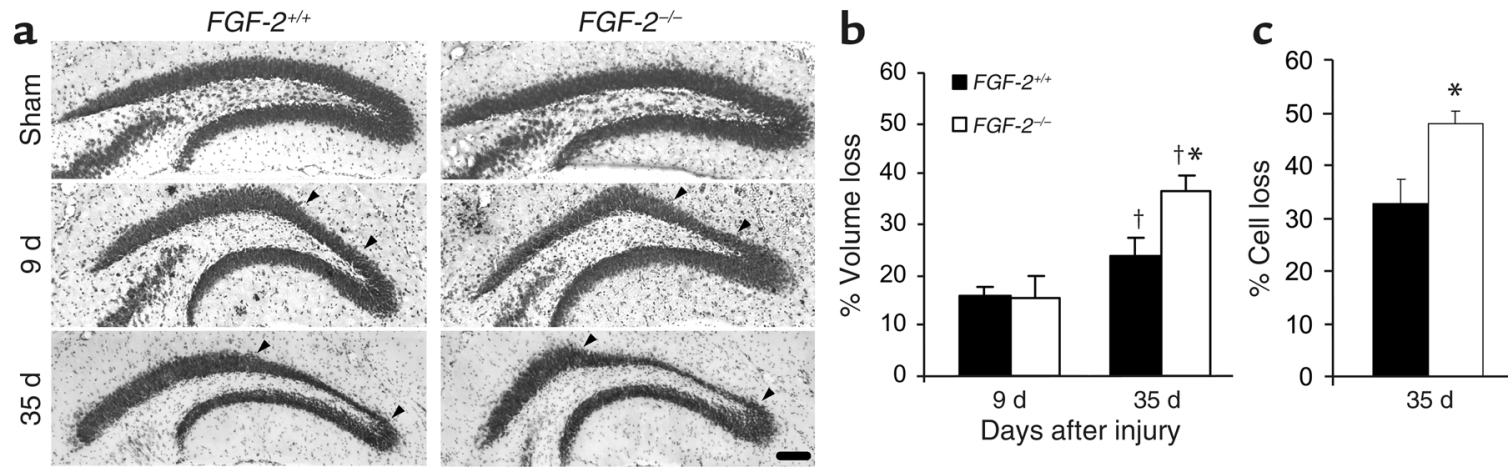

\section{Figure 4}

Cell loss in ipsilateral GCL of DG after $\mathrm{CCl}$ in $\mathrm{FGF}-2^{+/+}$and $\mathrm{FGF}-2^{-/-}$mice. (a) Mice were killed on either day 9 or day 35 after CCl. Coronal brain sections were obtained at bregma $-2.5 \mathrm{~mm}$ (cresyl violet staining). In sham-operated animals, there were no differences in DG morphology between $\mathrm{FGF}-2^{+/+}$and $\mathrm{FGF}-2^{-/-}$, and no apparent cell loss was observed at 35 days (upper panels). By 9 days after $\mathrm{CCl}$, cell loss was apparent in the superomedial part of the upper blade of the DG in both $F G F-2^{+/+}$and $F G F-2^{-/-}$mice (arrowheads, middle panels). By 35 days, cell loss was more prominent in FGF-2-/- mice (arrowheads, lower panels). Scale bar: $100 \mu \mathrm{m}$. Changes in volume (b) and cell loss (c) in GCL of DG after $\mathrm{CCl}$ in $\mathrm{FGF}-2^{+/+}$and $\mathrm{FGF}-2^{-/-}$mice. Mice were killed at either 9 or 35 days after $\mathrm{CCl}$, and coronal brain sections were stained with cresyl violet. The GCL volume in the ipsilateral DG was expressed as a percentage of volume measured in the corresponding region within the contralateral DG (see Methods). The percentage change in the number of neurons within GCL in the ipsilateral DG was calculated relative to that of the contralateral side (see Methods). GCL volume and neuron numbers were significantly decreased in $\mathrm{FGF}-2^{-/-}$mice (white bars) compared with $\mathrm{FGF}-2^{+/+}$mice (black bars) 35 days after injury. ${ }^{\dagger} P<0.05$ compared with mice of same genotype 9 days after $\mathrm{CCl}$. ${ }^{*} P<0.05$ compared with $F G F-2^{+/+}$mice on the same day after injury ( $n=4-7$ per group).

\section{Discussion}

TBI causes loss of volume within the GCL of DG (29). In this study, the volume decreased in both $\mathrm{FGF}^{-2^{-/}}$ and $\mathrm{FGF}_{-2} 2^{++}$mice, with $\mathrm{FGF}-2^{-/-}$mice suffering a greater decrease by 35 days posttrauma (Figure 4, Table 3). Furthermore, this volume loss corresponded to decreased numbers of granule cells, again with greater cell loss in $\mathrm{FGF}_{-2 /-}$ mice (Table 3). We found that enhancing expression of FGF-2 preserved the integrity of GCL after TBI. There are at least two possible mechanisms: Production of new cells and reduction of cell loss over time (27-30). Both may be operative in these experiments. We counted the number of cells in the GCL in FGF-2-/- and WT mice (Table 2). Thirty thousand fewer cells were lost in $\mathrm{FGF}^{-2^{+/+}}$animals compared with $F G F-2^{-/-}$mice. This would suggest that basal FGF-2 levels expressed in the WT can be neuroprotective. Interestingly, differences in TUNEL staining were not detected between groups 9 and 35 days after TBI, despite these group differences in cell loss. In addition, FGF-2 promoted neurogenesis in injured brain because 1100 more newly born neurons were found in $\mathrm{FGF}-2^{+/+}$animals at 35 days after injury, whereas only 600 new neurons were detected in the KO. Thus, our data suggest that endogenously generated FGF-2 regulates cell populations through manipulating neurogenesis as well as attenuating neurodegeneration in the GCL after TBI.

The dual role of FGF-2 was also confirmed through gene transfer experiments. Increased levels of FGF-2 enhanced cell division in the SGZ by 2.2-fold compared with control vector (Figure 2). At 5 weeks after TBI, FGF-2 vector yielded 2.5 -fold more newborn granule neurons compared with the control vector (Table 2), indicating that newly generated precursor cells proceed on to neuronal differentiation after FGF-2 gene transfer. Thus, neuronal production can be enhanced through upregulation of progenitor cell proliferation in the GCL after TBI by vector-mediated FGF-2 gene delivery. Moreover, posttraumatic overexpression of FGF-2 reduced the GCL volume loss by $10 \%$ (day 35 ) compared with animals injected with control vector (Figure 5). These results suggest that acute treatment with HSV-1 amplicon vectors expressing FGF-2 after the onset of TBI can protect against the chronic, progressive volume loss in ipsilateral DG that occurs over

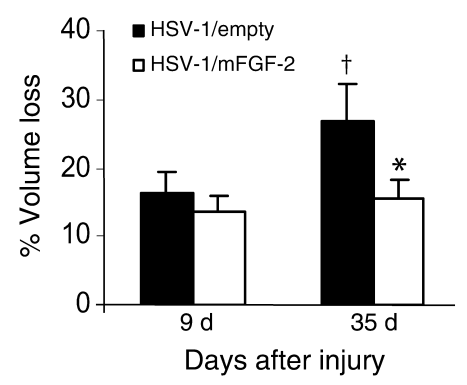

Figure 5

Quantification of volume loss of GCL in C57BL/6 mice injected with $\mathrm{HSV}-1 / \mathrm{mFGF}-2$ or HSV-1/empty after $\mathrm{CCl}$. Mice were injected with HSV-1/mFGF-2 or HSV-1/empty 1 hour after $\mathrm{CCl}$ and analyzed on day 35 . The volume in the ipsilateral GCL was expressed as a percentage of volume occupied by the corresponding region in the contralateral DG (see Methods). HSV-1/mFGF-2 (white bars) attenuated the volume loss compared with treatment with HSV-1/empty (black bars). ${ }^{\dagger} P<0.05$ compared with mice (HSV-1/empty) on day 9 after $\mathrm{CCl}$. ${ }^{*} P<0.05$ compared with HSV-1/empty group on day 35 after injury ( $n=6$ per group). 
several weeks after CCI. Furthermore, FGF-2 contributes to DG integrity after TBI by upregulating neurogenesis, based on the distribution pattern of newborn neurons labeled with BrdU. The total number of BrdU-labeled cells increased by gene transfer of FGF-2, and these cells migrated into the granule layer of DG and accumulated around the thinned part of the DG at day 35 (Figure 3), presumably contributing to attenuation of volume loss of GCL at 35 days after TBI.

Although SGZ cell division was promoted after TBI compared with sham-operated mice (Figure 1b), FGF-2 levels in the ipsilateral hippocampus did not change after TBI. Normally, FGF-2 does not have a signal sequence for cell secretion through the endoplasmic reticulum/Golgi apparatus $(47,48)$, and it is probably released extracellularly only after cell damage. According to this hypothesis, it is speculated that FGF-2 plays a negligible role in adult neurogenesis in the normal state, and factors other than FGF-2 must contribute to regulation of proliferation and differentiation of neural progenitor cells in the DG. In fact, under basal conditions, there was no difference in dividing progenitor populations between $\mathrm{FGF}_{-2 /+}$ and $\mathrm{FGF}^{+2^{-/-}}$ mice (Figure 1b), despite differences in FGF-2 levels in the hippocampus (15). With respect to FGF-2 release, plasminogen activator-mediated proteolysis provides a mechanism for dissociation of biologically active FGF-2-heparan sulfate complexes from the extracellular matrix, rendering FGF-2 more biologically active (47-49). Recently, TBI was shown to activate plasminogen activator $(50,51)$. We therefore speculate that TBI may promote FGF-2 dissociation from extracellular matrix and release from damaged cells. This phenomenon may account for the increase seen in SGZ cell division in $\mathrm{FGF}_{-2 /+}$ mice after TBI without measurable differences in FGF-2 levels from basal condition. Importantly, gene transfer of FGF-2 with an engineered secretion signal further enhanced the SGZ cell division in the injured DG with an increase in FGF-2 levels (Figure 2e). Thus, control of FGF-2 expression as well as its cellular release appears to be critically important in the regulation of progenitor cell proliferation after brain injury. In addition, injured tissue produces other factor(s) that convert latent FGF-2 to an active form $(49,52)$, consistent with a recent report suggesting that endogenous stimulatory signals are required for the action of growth factors potentiating ischemiainduced neurogenesis (31).

In conclusion, we have shown that overexpression of FGF-2 increased neurogenesis in the adult hippocampus after TBI, whereas neurogenesis was reduced in FGF-2-deficient mice. The results obtained suggest that FGF-2 is, at least in part, responsible for regulating neuronal replacement, as well as attenuating neuronal loss after TBI. FGF-2 supplementation may provide a rational strategy to treat brain injury by simultaneously enhancing neurogenesis and reducing neurodegeneration. Insights into the functions of FGF-2, together with further understanding about other factors regulating regeneration and protection, should provide a strategy for repair of CNS injury after trauma, and for other CNS injuries and disorders, such as cerebral ischemia and neurodegenerative diseases.

\section{Acknowledgments}

We thank S.P. Finklestein and H. Sugimori for their help with the breeding of KO mice; P. Bhide, T. Mitsuhashi, and Y. Wu for their technical advice; R. Russo and $\mathrm{S}$. McDavitt for editorial assistance on this article. This work was supported by NIH Interdepartmental Stroke Program Project 5 P50 NS10828 (M.A. Moskowitz), NINDS grant NS24279 (X.O. Breakefield). S. Yoshimura and Y. Takagi were supported by the Japan Society for the Promotion of Science fellowships, and M.J. Whalen by NIH KO8 NS41969-02.

1. Altman, J., and Das, G.D. 1965. Autoradiographic and histological evidence of postnatal hippocampal neurogenesis in rats. J. Comp. Neurol. 124:319-335.

2. Eriksson, P.S., et al. 1998. Neurogenesis in the adult human hippocampus. Nat. Med. 4:1313-1317.

3. Gage, F.H. 2000. Mammalian neural stem cells. Science. 287:1433-1438.

4. Gould, E., Tanapat, P., McEwen, B.S., Flugge, G., and Fuchs, E. 1998. Proliferation of granule cell precursors in the dentate gyrus of adult monkeys is diminished by stress. Proc. Natl. Acad. Sci. U. S. A. 95:3168-3171.

5. Kempermann, G., Kuhn, H.G., and Gage, F.H. 1997. More hippocampal neurons in adult mice living in an enriched environment. Nature. 386:493-495.

6. Kempermann, G., Kuhn, H.G., and Gage, F.H. 1997. Genetic influence on neurogenesis in the dentate gyrus of adult mice. Proc. Natl. Acad. Sci. U. S. A. 94:10409-10414.

7. Kornack, D.R., and Rakic, P.C. 1999. Continuation of neurogenesis in the hippocampus of the adult macaque monkey. Proc. Natl. Acad. Sci. U. S. A. 96:5768-5773.

8. Covolan, L., Ribeiro, L.T., Longo, B.M., and Mello, L.E. 2000. Cell damage and neurogenesis in the dentate granule cell layer of adult rats after pilocarpine- or kainate-induced status epilepticus. Hippocampus. 10:169-180.

9. Dash, P.K., Mach, S.A., and Moore, A.N. 2001. Enhanced neurogenesis in the rodent hippocampus following traumatic brain injury. J. Neurosi. Res. 63:313-319.

10. Gould, E., and Tanapat, P. 1997. Lesion-induced proliferation of neuronal progenitors in the dentate gyrus of the adult rat. Neuroscience. 80:427-436.

11. Gray, W.P., and Sundstrom, L.E. 1998. Kainic acid increases the proliferation of granule cell progenitors in the dentate gyrus of the adult rat. Brain Res. 790:52-59.

12. Jin, K., et al. 2001. Neurogenesis in dentate subgranular zone and rostral subventricular zone after focal cerebral ischemia in the rat. Proc. Natl. Acad. Sci. U. S. A. 98:4710-4715.

13. Liu, J., Solway, K., Messing, R.O., and Sharp, F.R. 1998. Increased neurogenesis in the dentate gyrus after transient global ischemia in gerbils. J. Neurosci. 18:7768-7778.

14. Takagi, Y., et al. 1999. Proliferation of neuronal precursor cells in the dentate gyrus is accelerated after transient forebrain ischemia in mice. Brain Res. 831:283-287.

15. Yoshimura, S., et al. 2001. FGF-2 regulation of neurogenesis in adult hippocampus after brain injury. Proc. Natl. Acad. Sci. U. S. A. 98:5874-5879.

16. Cameron, H.A., Hazel, T.G., and McKay, R.D. 1998. Regulation of neurogenesis by growth factors and neurotransmitters. J. Neurobiol. 36:287-306.

17. Ghosh, A., and Greenberg, M.E. 1995. Distinct roles for bFGF and NT-3 in the regulation of cortical neurogenesis. Neuron. 15:89-103.

18. Kuhn, H.G., Winkler, J., Kempermann, G., Thal, L.J., and Gage, F.H. 1997. Epidermal growth factor and fibroblast growth factor-2 have different effects on neural progenitors in the adult rat brain. J. Neurosci. 17:5820-5829.

19. Temple, S., and Qian, X. 1995. bFGF, neurotrophins, and the control or cortical neurogenesis. Neuron. 15:249-252.

20. Vicario-Abejon, C., Johe, K.K., Hazel, T.G., Collazo, D., and McKay, R.D. 1995. Functions of basic fibroblast growth factor and neurotrophins in the differentiation of hippocampal neurons. Neuron. 15:105-114.

21. Vaccarino, F.M., et al. 1999. Changes in cerebral cortex size are governed by fibroblast growth factor during embryogenesis. Nat. Neurosci. 2:246-253. 
22. Ray, J., Peterson, D.A., Schinstine, M., and Gage, F.H. 1993. Proliferation, differentiation, and long-term culture of primary hippocampal neurons. Proc. Natl. Acad. Sci. U. S. A. 90:3602-3606.

23. Pettmann, B., Labourdette, G., Weibel, M., and Sensenbrenner, M. 1986. The brain fibroblast growth factor (FGF) is localized in neurons. Neurosi. Lett. 68:175-180.

24. Walicke, P.A., Feige, J.J., and Baird, A. 1989. Characterization of the neuronal receptor for basic fibroblast growth factor and comparison to receptors on mesenchymal cells. J. Biol. Chem. 264:4120-4126.

25. Wanaka, A., Johnson, E.M., Jr., and Milbrandt, J. 1990. Localization of FGF receptor mRNA in the adult rat central nervous system. Neuron. 5:267-281.

26. Ku, P.T., and D'Amore, P.A. 1995. Regulation of basic fibroblast growth factor (bFGF) gene and protein expression following its release from sublethally injured endothelial cells. J. Cell Biochem. 58:328-343.

27. Cuevas, P. 1997. Therapeutic prospects for fibroblast growth factor treatment of brain ischemia. Neurol. Res. 19:355-356.

28. Dietrich, W.D., Alonso, O., Busto, R., and Finklestein, S.P. 1996. Posttreatment with intravenous basic fibroblast growth factor reduces histopathological damage following fluid-percussion brain injury in rats. J. Neurotrauma. 13:309-316.

29. Mocchetti, I., and Wrathall, J.R. 1995. Neurotrophic factors in central nervous system trauma. J. Neurotrauma. 12:853-870.

30. Tretter, Y.P., et al. 2000. Induction of activin A is essential for the neuroprotective action of basic fibroblast growth factor in vivo. Nat. Med. 6:812-815.

31. Nakatomi, H., et al. 2002. Regeneration of hippocampal pyramidal neurons after ischemic brain injury by recruitment of endogenous neural progenitors. Cell. 110:429-441.

32. Colicos, M.A., and Dash, P.K. 1996. Apoptotic morphology of dentate gyrus granule cells following experimental cortical impact injury in rats: possible role in spatial memory deficits. Brain Res. 739:120-131.

33. Dietrich, W.D., Alonso, O., and Halley, M. 1994. Early microvascular and neuronal consequences of traumatic brain injury: a light and electron microscopic study in rats. J. Neurotrauma. 11:289-301.

34. Scheff, S.W., Baldwin, S.A., Brown, R.W., and Kraemer, P.J. 1997. Morris water maze deficits in rats following traumatic brain injury: lateral controlled cortical impact. J. Neurotrauma. 14:615-627.

35. Smith, D.H., et al. 1995. A model of parasagittal controlled cortical impact in the mouse: cognitive and histopathologic effects. J. Neurotrauma. 12:169-178.

36. Zhou, M., et al. 1998. Fibroblast growth factor 2 control of vascular tone. Nat. Med. 4:201-207.

37. Steward, O., et al. 1999. Genetic approaches to neurotrauma research: opportunities and potential pitfalls of murine models. Exp. Neurol. 157:19-42.

38. Whalen, M.J., et al. 1999. Reduction of cognitive and motor deficits after traumatic brain injury in mice deficient in poly(ADP-ribose) polymerase. J. Cereb. Blood Flow Metab. 19:835-842.

39. Ikeda, K., Saeki, Y., Gonzalez-Agosti, C., Ramesh, V., and Chiocca, E.A. 1999. Inhibition of NF2-negative and NF2-positive primary human meningioma cell proliferation by overexpression of merlin due to vectormediated gene transfer. J. Neurosurg. 91:85-92.

40. Fraefel, C., et al. 1996. Helper virus-free transfer of herpes simplex virus type 1 plasmid vectors into neural cells. J. Virol. 70:7190-7197.

41. Cavalieri, B. 1966. Geometria degli indivisibli. Unione Tipografico-Editrice. Torino, Italy. $872 \mathrm{pp}$.

42. Nakamura, M., et al. 1999. Overexpression of Bcl-2 is neuroprotective after experimental brain injury in transgenic mice. J. Comp. Neurol. 412:681-692.

43. Takeuchi, A., et al. 2000. Age-related amyloid beta deposition in transgenic mice overexpressing both Alzheimer mutant presenilin 1 and amyloid beta precursor protein Swedish mutant is not associated with global neuronal loss. Am. J. Pathol. 157:331-339.

44. West, M.J., Slomianka, L., and Gundersen, H.J. 1991. Unbiased stereological estimation of the total number of neurons in the subdivisions of the rat hippocampus using the optical fractionator. Anat. Rec. 231:482-497.

45. West, M.J. 1993. New stereologic methods for counting neurons. Neurobiol. Aging. 14:275-285.

46. Rink, A., et al. 1995. Evidence of apoptotic cell death after experimental traumatic brain injury in the rat. Am. J. Pathol. 147:1575-1583.

47. Friesel, R.E., and Maciag, T. 1995. Molecular mechanisms of angiogenesis: fibroblast growth factor signal transduction. FASEB J. 9:919-925.

48. Saksela, O., and Rifkin, D.B. 1990. Release of basic fibroblast growth factor-heparan sulfate complexes from endothelial cells by plasminogen activator-mediated proteolytic activity. J. Cell Biol. 110:767-775.

49. Penc, S.F., et al. 1998. Dermatan sulfate released after injury is a potent promoter of fibroblast growth factor-2 function. J. Biol. Chem. 273:28116-28121.

50. Dietzmann, K., et al. 2000. Expression of the plasminogen activator system and the inhibitors PAI- 1 and PAI- 2 in posttraumatic lesions of the CNS and brain injuries following dramatic circulatory arrests: an immunohistochemical study. Pathol. Res. Pract. 196:15-21.

51. Reilly, J.F., and Kumari, V.G. 1996. Alterations in fibroblast growth factor receptor expression following brain injury. Exp. Neurol. 140:139-150.

52. Kato, M., et al. 1998. Physiological degradation converts the soluble syndecan-1 ectodomain from an inhibitor to a potent activator of FGF-2. Nat. Med. 4:691-697. 